\title{
3D Gaze Estimation using Eye Vergence
}

\author{
Esteban Gutierrez Mlot $^{1}$, Hamed Bahmani ${ }^{2}$, Siegfried Wahl ${ }^{2}$ and Enkelejda Kasneci ${ }^{1}$ \\ ${ }^{1}$ Perception Engineering Group, Computer Science Department, University of Tübingen, \\ Sand 14, 72076 Tübingen, Germany \\ ${ }^{2}$ ZEISS Vision Science Lab, Institute for Ophthalmic Research, University of Tübingen, \\ Röntgenweg 11, 72076 Tübingen, Germany
}

Keywords: $\quad 3 D$ Calibration, Eye Tracking, Eye Vergence, Gaze Localization, Depth Perception.

\begin{abstract}
We propose a fast and robust method to estimate the 3D gaze position based on the eye vergence information extracted from eye-tracking data. This method is specially designed for Point-of-Regard (PoR) estimation in non-virtual environments with the aim to make it applicable to the study of human visual attention deployment in natural scenarios. Our approach starts with a calibration step at different depth distances in order to achieve the best depth approximation. In addition, we investigate the distance range, for which state-of-the-art eyetracking technology allows 3D gaze estimation based on eye vergence. Our method provides a mean accuracy of $1.2^{\circ}$ at a working distance between $200 \mathrm{~mm}$ and $400 \mathrm{~mm}$ from the user without requiring calibrated lights or cameras.
\end{abstract}

\section{INTRODUCTION}

Determining the 3D gaze position in natural settings is of great interest for different fields of research and applications, e.g., investigation human attention deployment when buying products (Gidlöf et al., 2013) or analysis of visual behavior for driver assistance in the automotive industry (Braunagel et al., 2015; Fletcher et al., 2005; Kasneci et al., 2014; Kasneci et al., 2015). Moreover, in virtual environments, depth estimation could help to improve the sensation of immersion into the virtual world optimizing the level of detail (Duchowski, 2007) or adjusting the sharpness (Hillaire et al., 2008), where the user's attention is focused. In this paper, we address a central question for video-based 3D gaze estimation, i.e., how much focus depth information can be derived solely from images of the user's eyes.

3D gaze estimation in video-based eye tracking consists of mapping the pupil's center estimate extracted from the eye image to an actual 3D position in the scene. For this purpose, depth perception has to be extracted from the visual system. The human brain uses several sources of information for 3D vision and reconstruction including monocular cues, such as occlusion, as well as binocular disparity (Kandel et al., 2000). Furthermore, it receives information from three oculomotor systems to perceive depth (Reichelt et al., 2010): accommodation, miosis, and vergence.
The sense of depth is delivered to the viewer by any of these depth cues. Accommodation is the process by which the vertebrate eye changes optical power to maintain focus on an object as its distance varies (Davson, 2012). Miosis is the constriction of the pupil relative to the amount of light the pupil receives (Rogers, 1988). Finally, vergence is the simultaneous movement of both eyes in opposite directions to obtain or maintain single binocular vision (Cassin et al., 1984). For the purpose of designing a 3D eye-tracking system, measuring accommodation needs perfectly controlled conditions and complex devices (e.g., Powerrefractor $^{\mathrm{TM}}$ (Schaeffel et al., 1993)), or ultrasound biomicroscopy (Kasthurirangan, 2014); and it is not recommended for continuous long exposure. Miosis is easily measurable, but is very sensitive to ambient light (Cheng et al., 2006). Thus, among the three information sources from the oculomotor system, we are left with the vergence information to estimate the gaze position or Point of Regard $(P O R)$. In videobased 3D eye-tracking systems, the vergence information is the only signal that is robust and easy to measure.

Existing 3D gaze estimation techniques can be divided in two main categories: (i) methods based on a geometrical models of the eye, and (ii) methods based on interpolation, i.e., the direct mapping of eye position and $P O R$.

Common ground in the model-based approach 
is the use of multiple light sources to calculate the corneal center (Shih and Liu, 2004). Additional improvements allow restricted head movements (Craig Hennessey, 2009). Gaze estimation techniques in this category have a good accuracy but rely on multiple infrared lights pointing to the eye, which can be impractical in outdoor environments or unhealthy under certain conditions (Mulvey et al., 2008), (Kourkoumelis and Tzaphlidou, 2011). Furthermore, other algorithms do not require calibrated lighting features (Świrski and Dodgson, 2013), but there is no evidence for their application to non-virtual environments.

Approaches based on interpolation originate from 2D PoR estimation methods and are extended for binocular gaze vector estimation in 3D PoR (Duchowski et al., 2001). Other variations use neural networks instead of polynomial functions for a better accuracy (Essig et al., 2006) but are designed specifically for 3D displays. Neural networks are compared with a geometry-based algorithm, which provides better results for real-time execution (Wang et al., 2014). However, both methods have been only tested in virtual environments.

In summary, all previously mentioned methods lack the applicability in real-time, non-virtual, and non-laboratory scenarios. In this paper, we propose an online 3D gaze estimation method based on interpolation that is suitable for $3 \mathrm{D}$ eye-tracking in realworld scenarios. How the theoretical vergence angle varies with the observation distance to a target object is analyzed in Section 2.1. Based on this theoretical analysis we derive the range in which an eye tracker can determine the vergence angle with sufficient accuracy. The proposed 3D PoR estimation is presented in Section 2.2 with details regarding pupil position calculation explained in Section 2.2.1. The experimental validation of the method is described in Section 3.1 and results are presented in Section 3.2. Section 4 concludes this work.

\section{METHOD}

Our method determines a 3D gaze point from images of both eyes of the observer. After a calibration phase, the gaze position can be estimated. For the initial calibration step, several points on different depth planes are presented to the observer and looked at sequentially. While the observer is looking at a given point, the corresponding pupil center position is recorded. Pupil center and point position are then used to calculate the gaze vector from both eyes and then estimate the vergence angle. At the end of this step, information about the relationship between the vergence angle and distance is calculated and stored for later use. For the final estimation step, the vergence angle is calculated from the pupil centers and a $3 D P o R$ is calculated as described below.

\subsection{Relationship between Vergence and Observation Distance}

The vergence angle change is the difference between the vergence angles resulting when moving a target at given distance from the observer (see Figure 1) (Healy and Proctor, 2003). This change will be higher the closer the fixated object is to the observer. Within the range of 2 meters, the change in vergence angle decreases exponentially with distance (Howard, 2012) (see Figure 2), implying thus, the existence of a certain distance range in which the vergence angle change is measurable within an acceptable experimental error. Therefore, before applying the proposed 3D PoR estimation method, it is essential to find out within which range the eye-tracking signal can be used to extract useful information on vergence.

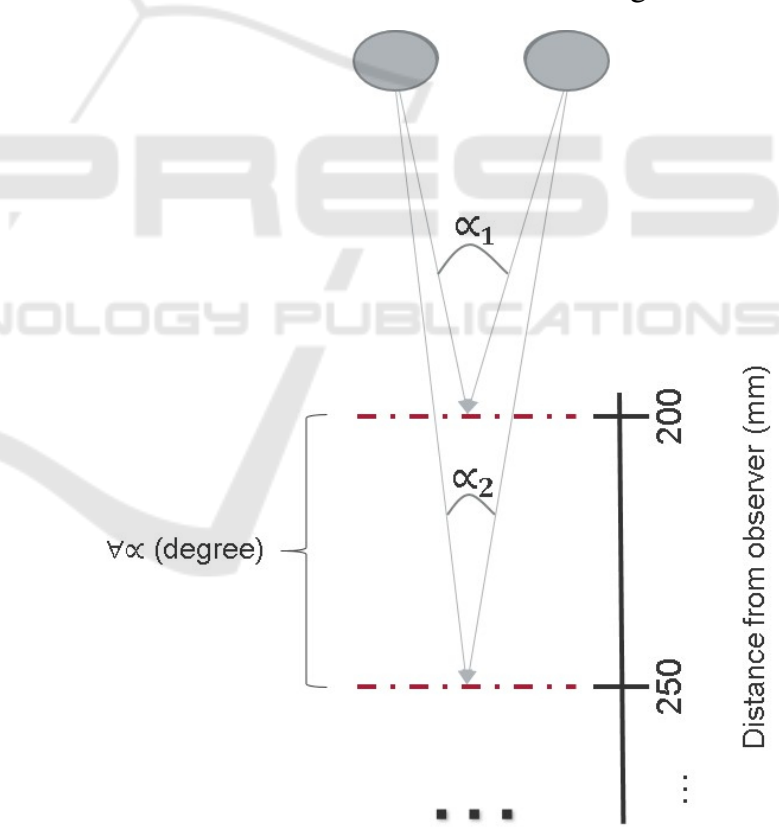

Figure 1: Calculation of the vergence angle difference.

To approach this, we first calculate the theoretical variation of the vergence angle with the distance. The relevant parameters are the positions of the eyes and the attended point in front of them. A ray is traced from each eye towards this point. For our theoretical considerations we assume that the fixated at point is positioned straight ahead of the eyes (from the center point between both eyes). Since the vergence angle depends on the distance to the eyes, other points on a 
sphere around the eyes would yield a similar result.

We sampled the vergence angle difference between planes positioned in steps of $50 \mathrm{~mm}$. The difference was calculated by subtracting the vergence angle value in a plane to the previous one, starting at the plane located further away from the observer. We can therefore infer the maximum distance at which the vergence difference between two sequential planes is still larger than our assumed eye-tracker accuracy. In consequence, planes with less vergence difference among them will be indistinguishable. For these calculations, we considered an interpupillary distance of $64 \mathrm{~mm}$, which correspond to the population mean (Dodgson, 2004).

Figure 2 shows the vergence angle for distances ranging from $20 \mathrm{~mm}$ to $1200 \mathrm{~mm}$ from the user. Measuring differences of less than $50 \mathrm{~mm}$ from $400 \mathrm{~mm}$ distance to the user requires a maximum error of $1^{\circ}$. This is the maximum distance limit we have chosen for the experiments, since the required accuracy is feasible. We use a minimum distance of $200 \mathrm{~mm}$ because looking at closer objects is very uncomfortable and these objects are hard to fixate. Moreover, this theoretical calculation has been corroborated by the empirical results obtained in our experiments.

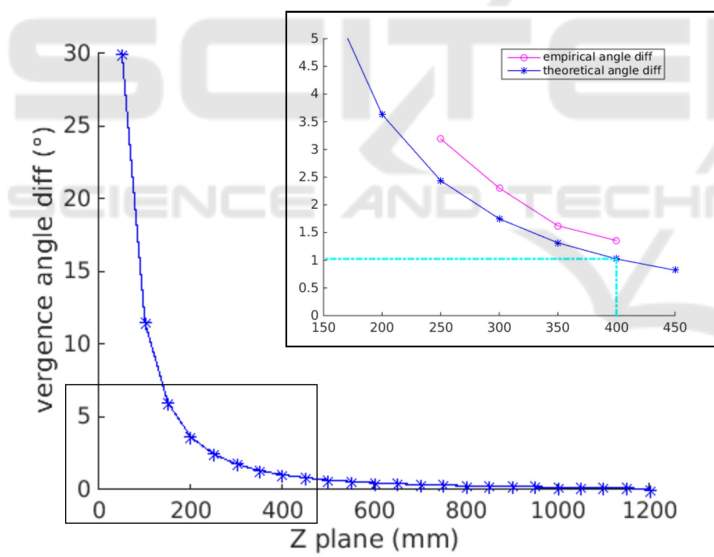

Figure 2: Theoretical angle difference of vergence and comparison with empirical values in setup range.

\subsection{Estimation of 3D Gaze Position}

The core idea of the proposed method is to cross the gaze rays of both eyes and thereby determine the 3D gaze position. Two steps have to be solved first: we have to determine the gaze ray of each eye and we have to find a solution to non-intersecting rays (which in 3D space will be more common than actually intersecting rays).

To solve the first issue we employ an interpolation-based approach as described in Section 2.2.2. To address the second problem, we estimate the intersection as the middle of the perpendicular line between the two rays, or the point where the interlinear distance is minimal.

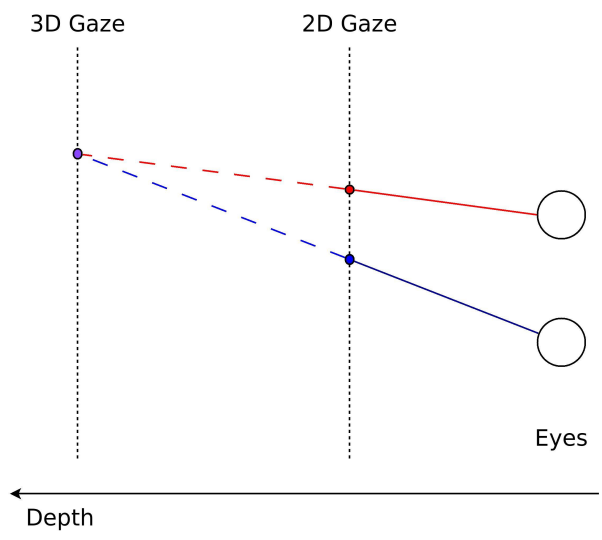

Figure 3: Ray trace from eyes to PoR.

Two different algorithms have been tested for $P o R$ estimation using the line of sight. The first one was proposed by (Wang et al., 2014), while the second approach is a pure geometrical procedure based on the Dan Sunday algorithm (Sunday, 2012). Despite being based on the same principles, the two methods perform differently. Best results have been achieved with the method proposed by (Wang et al., 2014).

Based on (Wang et al., 2014), we propose an algorithm that follows a two-step approach to estimate an accurate $3 \mathrm{D}$ gaze position in non-virtual environments. In a first step, a coarse depth approximation is done. The second step refines the 3D PoR in order to get the most accurate result possible. Both steps use triangulation mappings based on (Wang et al., 2014). However, the method presented in this paper differs at several steps:

- Our method is able to use calibration points from different depth layers. Therefore, we are able to calibrate not only in one plane but also the depth layer and to include subject-specific effects.

- We apply the method to an experimental dataset in a real-world environment, outside of a virtual reality and without stereoscopic displays.

- The polynomial mapping function as well as the pupil center detection of the first algorithm step is included in our procedure and not provided by a commercial eye-tracking software. Therefore, we can apply a custom mapping function and have full control over the estimation process.

- We do not rely on smoothing filters and can therefore shorten the execution time of the algorithm.

Since experimental measurements have shown that using only one plane for getting the interpolated 
points to calculate the line of sight $(L o S)$ is not accurate enough, we have used different base planes for interpolation. Additionally, error rises accordingly as the $P o R$ lies further away from the interpolation plane (see Figure 4). This problem is solved by selecting the closest interpolation plane for calculating PoR. In the first step, we used the first interpolation plane to get an approximate vergence angle. This vergence angle is used with a pre-calculated table (see Table 1) to select the closest interpolation plane to the PoR. Then the selected interpolation plane is used to calculate an accurate $P o R$ position in $3 \mathrm{D}$.

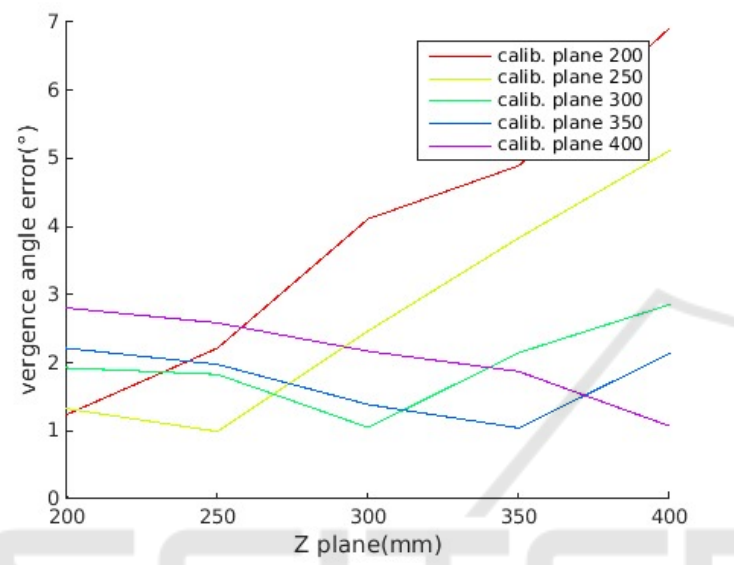

Figure 4: Vergence angle error using different calibration planes.

Table 1 is determined in a calibration procedure. This procedure consists of calculating the median vergence angle of 16 calibration points for each depth plane. All the points are on the selected plane (see Figure 7) and the vergence angles are calculated for each point using the eye positions and the target point.

Table 1: Relationship between eye vergence and viewing distance.

\begin{tabular}{cc}
\hline \hline Distance $(\mathrm{mm})$ & Vergence Angle $\left(^{\circ}\right)$ \\
\hline \hline 200 & 14.4 \\
250 & 11.6 \\
300 & 9.3 \\
350 & 7.4 \\
400 & 6.1 \\
\hline \hline
\end{tabular}

\subsubsection{Pupil Position Calculation}

In order to test the accuracy of the calibration method and to reduce other error sources, pupil center positions were determined by the ExCuSe algorithm (Fuhl et al., 2015a) first and checked manually afterwards (see Figure 5).

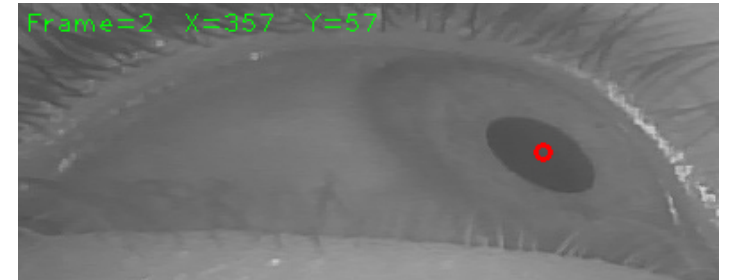

Figure 5: Pupil position detected by the custom developed program.

\subsubsection{Interpolation Function}

Interpolation is one of the fundamental steps used in feature-based gaze estimation (Chennamma and Yuan, 2013). The basis of this method is to obtain a polynomial function that relates the pupil position in the eye image to a position on a screen or a plane in space. The polynomial coefficients are approximated using a regression analysis. We tested different polynomial mapping functions. A $2^{\text {nd }}$ order polynomial function (see Equation 1) showed best results and is therefore used in this work. This is consistent with a study by (Cerrolaza et al., 2012) that concludes that higher order polynomials do not improve the system behavior.

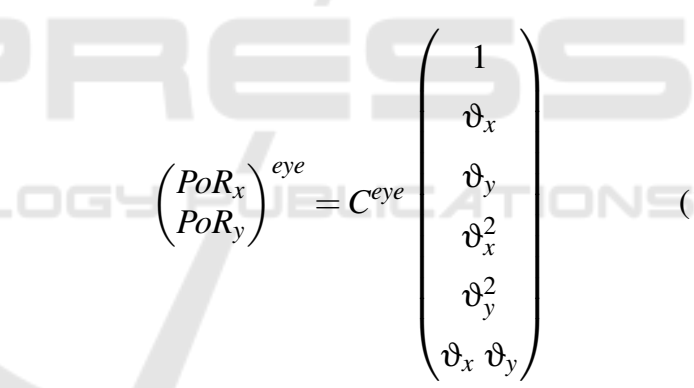

where $P o R_{x}$ and $P o R_{y}$ are the interpolated points in the plane of one eye, $\vartheta_{x}$ and $\vartheta_{y}$ are the pupil center position of an eye in the image and $C^{e y e}$ is the coefficients matrix for this eye.

\section{EXPERIMENTAL EVALUATION}

\subsection{Setup}

To corroborate our method for gaze estimation, an experimental setup has been developed. The aim of this setup is to provide empirical validation of $P O R$ estimation in a real environment only by using the vergence angle information. The test setup comprises a chin rest for fixing the subject's head position, a moving actuator for changing depth plane $(Z$ plane) and a head-mounted camera device (see Fig- 

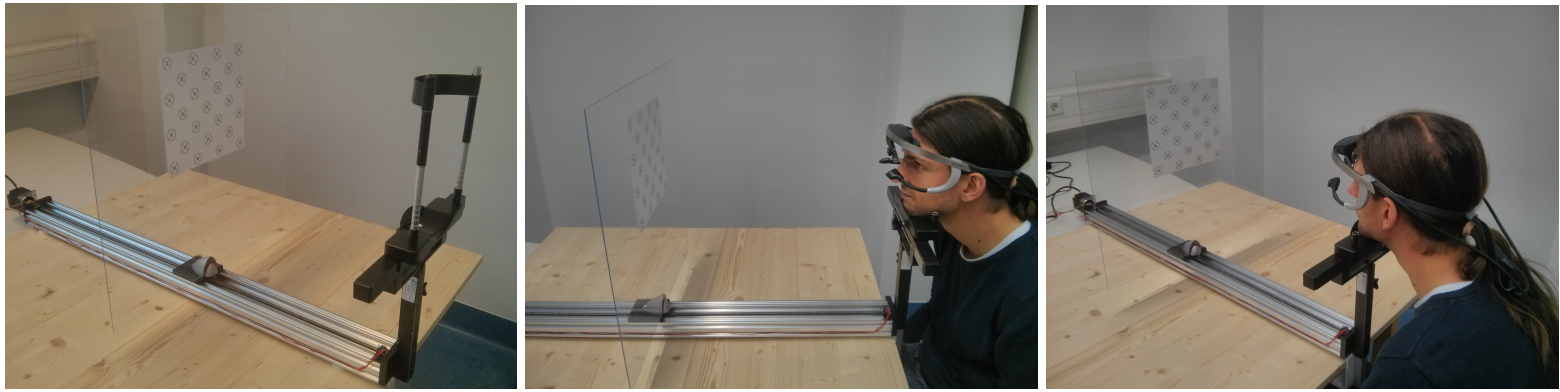

Figure 6: Experimental setup for calibration and evaluation. The complete setup is transportable and consists of a camera and a motorized linear rail system for moving the target.

ure 6). The moving actuator supports a transparent Plexiglas ${ }^{\circledR}$ panel with 25 marker points (see Figure 7).

The test procedure consisted of asking the subject to look at specific points on the panel (numbered 1 to 25) at different depths. The points were separated $3 \mathrm{~cm}$ from each other covering an area of $20 \times 20$ $\mathrm{cm}^{2}$. The central point was located in the middle between the subject's eyes. The Plexiglas ${ }^{\circledR}$ plane was presented at 20,25,30, 35 and $40 \mathrm{~cm}$ distance from the subject. The full workspace covered volume of 20 $\times 20 \times 20 \mathrm{~cm}^{3}$. Later on, 16 points of the panel were used for calibration and all 25 points were used for evaluation of the algorithm (see Figure 7).

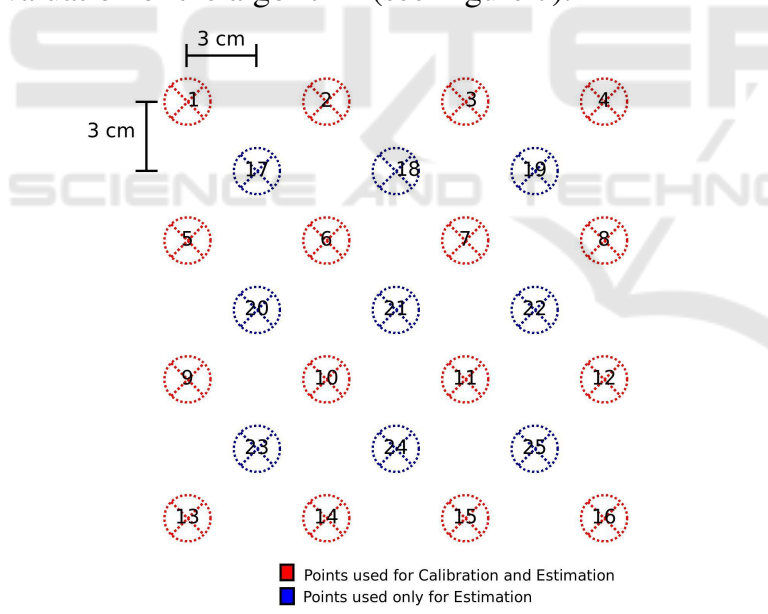

Figure 7: Grid pattern presented to the subjects. The red colored circles are used for calibration. Additionally the blue circles are used for evaluation.

During the experiment, images of the subject's eyes were taken in the exact time when the subject fixated a specific point. These images of $720 \times 480$ pixels resolution were used to calculate the pupil position.

Six subjects took part in the experiment. The age of participants varied between 22 and 34 years, recruited on a voluntary basis. All subjects have normal vision except for one, who was wearing corrective contact lenses, which did not affect the test.

\subsection{Results}

Table 2 represents the error between the estimated and the real position for all the points. Error for each axis, Euclidean distance and angular difference (see Equation 2) are shown for each Z plane. Furthermore, overall average and standard deviation from all planes are presented.

$$
\begin{gathered}
\text { Dist. }=\sqrt{\left(X_{r}-X_{e}\right)^{2}+\left(Y_{r}-Y_{e}\right)^{2}+\left(Z_{r}-Z_{e}\right)^{2}} \\
\operatorname{dot} p=X_{e} * X_{r}+Y_{e} * Y_{r}+Z_{e} * Z_{r} \\
\text { lenSq } q_{e}=X_{e}^{2}+Y_{e}^{2}+Z_{e}^{2} \\
\text { lenSq } q_{r}=X_{r}^{2}+Y_{r}^{2}+Z_{r}^{2} \\
\text { Angle }=\arccos \left(\frac{\text { dotp }}{\sqrt{\text { lenSqe } q_{e} \text { lenSqr }}}\right)
\end{gathered}
$$

where $r$ is real position and $e$ is estimated position.

The results show an average error of $1.2^{\circ}$, which lies within our expectations. As shown previously in Figure 2, this accuracy allows us to estimate $P o R$ in a distance below $400 \mathrm{~mm}$.

The accuracy of the proposed algorithm can be compared with a model-based method such as the one proposed by Hennessey (Craig Hennessey, 2009). Both algorithms estimate $P O R$ in non-virtual scenarios for planes positioned at $200-400 \mathrm{~mm}$ from the subject. The comparison is made only in terms of the distance error for each axis and Euclidean distance. The method proposed by Wang (Wang et al., 2014) employs 5 planes positioned at $380-620 \mathrm{~mm}$ from the subject, separated $6 \mathrm{~cm}$ from each other. It is difficult to infer precise values from the data shown in (Wang et al., 2014), therefore, we made a rough estimation of their results using the histogram bars provided. Table 3 presents a comparison of these three methods. As shown in Table 3, our method achieves significantly better accuracy, especially with respect to the $\mathrm{Z}$ axis. It is noticeable, that this level of accuracy is achieved without using additional infrared illumination and the experiments have been carried out 
Table 2: Average accuracy and standard deviation of 3D PoR estimation over the workspace for all subjects.

\begin{tabular}{cccccccc}
\hline \hline $\begin{array}{c}\text { Z Depth } \\
(\mathrm{mm})\end{array}$ & \multicolumn{2}{c}{ Average Accuracy $(\mathrm{mm})$} & $\begin{array}{c}\text { Average Accuracy } \\
\left({ }^{\circ}\right)\end{array}$ & \multicolumn{2}{c}{ Standard Deviation } \\
$(\mathrm{mm})$ & $\left({ }^{\circ}\right)$ \\
\hline \hline 200 & 3.5 & 4.3 & 9.1 & 10.8 & 1.2 & 8.6 & 0.6 \\
250 & 2.7 & 3.6 & 8.6 & 10.5 & 0.8 & 9.4 & 0.8 \\
300 & 2.3 & 3.1 & 10.4 & 13.3 & 1.2 & 7.4 & 0.8 \\
350 & 3.8 & 4.4 & 18.7 & 20.8 & 1.5 & 8.9 & 0.6 \\
400 & 2.4 & 3.7 & 14.5 & 15.9 & 1.2 & 8.5 & 0.5 \\
\hline Overall & 2.9 & 3.8 & 12.3 & 14.3 & 1.2 & 8.6 & 0.7 \\
\hline \hline
\end{tabular}

in a natural setting, in contrast to related approaches that use $3 \mathrm{D}$ displays.

Table 3: Average results for the five depth positions for the proposed method and state-of-the art as presented in (Craig Hennessey, 2009) and (Wang et al., 2014).

\begin{tabular}{cccccc}
\hline \hline $\begin{array}{c}\text { Method } \\
(\mathrm{mm})\end{array}$ & $\mathrm{X}$ & $\mathrm{Y}$ Avg. accuracy $(\mathrm{mm})$ & $\mathrm{Z}$ & Euc. & Std.dev. \\
$(\mathrm{mm})$
\end{tabular}

The proposed method enables a robust calculation of the gaze point in 3D as required in several application fields, e.g., in the context of assistive technology (especially where gesture and gaze is coupled for interaction (Lukic et al., 2014)) or for gaze-based interaction with multimedia in the car. In our future work, we will integrate the proposed method in operation microscopes in order to enable gaze-based autofocus. Thus, instead of manual focusing, the fixations locations of the surgeon will be analyzed in an online fashion (e.g., as in (Tafaj et al., 2012)) and coupled with the vergence information to determine the focus depth in an automated way. Despite the above use cases, the proposed method could improve current developments based on 2D eye tracking (Lopes et al., 2012) with detailed 3D gaze point selection.

\section{CONCLUSIONS}

We presented an accurate method for gaze point estimation in 3D based on the eye vergence calculated from eye-tracking data. Our evaluation in a natural setting with 6 subjects, 5 different depth planes, and a total number of 125 calibration points showed that our method achieves a high average accuracy of $1.2^{\circ}$. According to our experimental measurements, the upper limit for the viewing distance when less than $1^{\circ}$ accuracy is required, is $400 \mathrm{~mm}$ from the observer. Future work will include incorporating different pupil detection algorithms, e.g. (Fuhl et al.,
$2015 \mathrm{~b}$ ), and finding the optimum number of interpolation planes within the range $200-400 \mathrm{~mm}$.

\section{ACKNOWLEDGEMENTS}

This project has been founded by the Inter-University Center for Medical Technologies Stuttgart - Tübingen (IZST) and Carl Zeiss Meditec AG.

\section{REFERENCES}

Braunagel, C., Stolzmann, W., Kasneci, E., and Rosenstiel, W. (2015). Driver-activity recognition in the context of conditionally autonomous driving. In 2015 IEEE 18th International Conference on Intelligent Transportation Systems (ITSC), pages 1652-1657.

Cassin, B., Rubin, M. L., and Solomon, S. (1984). Dictionary of eye terminology. Triad Publishing Company.

Cerrolaza, J. J., Villanueva, A., and Cabeza, R. (2012). Study of Polynomial Mapping Functions in VideoOculography Eye Trackers. ACM Transactions on Computer-Human Interaction, 19(2):1-25.

Cheng, A. C., Rao, S. K., Cheng, L. L., and Lam, D. S. (2006). Assessment of pupil size under different light intensities using the procyon pupillometer. Journal of Cataract \& Refractive Surgery, 32(6):1015-1017.

Chennamma, H. and Yuan, X. (2013). A Survey on Eye-Gaze Tracking Techniques. arXiv preprint arXiv:1312.6410, 4(5):388-393.

Craig Hennessey, P. L. (2009). Noncontact binocular eyegaze tracking for point-of-gaze estimation in three dimensions. IEEE Transactions on Biomedical Engineering, 56(3):790-799.

Davson, H. (2012). Physiology of the Eye. Elsevier.

Dodgson, N. A. (2004). Variation and extrema of human interpupillary distance," in stereoscopic displays and virtual reality systems. In Proc. SPIE 5291, pages 3646.

Duchowski, A. T. (2007). Foveated Gaze-Contingent Displays for Peripheral LOD Management, 3D Visualization, and Stereo Imaging. 3(4).

Duchowski, A. T., Medlin, E., Gramopadhye, A., Melloy, B., and Nair, S. (2001). Binocular eye tracking in VR 
for visual inspection training. Proceedings of the ACM symposium on Virtual reality software and technology - VRST'01, page 1.

Essig, K., Pomplun, M., and Ritter, H. (2006). A neural network for 3D gaze recording with binocular eye trackers. International Journal of Parallel, Emergent and Distributed Systems, 21(February 2015):79-95.

Fletcher, L., Loy, G., Barnes, N., and Zelinsky, A. (2005). Correlating driver gaze with the road scene for driver assistance systems. Robotics and Autonomous Systems, 52(1):71-84.

Fuhl, W., Kübler, T. C., Sippel, K., Rosenstiel, W., and Kasneci, E. (2015a). Excuse: Robust pupil detection in real-world scenarios. In Azzopardi, G. and Petkov, N., editors, Computer Analysis of Images and Patterns - 16th International Conference, CAIP 2015, Valletta, Malta, September 2-4, 2015 Proceedings, Part I, volume 9256 of Lecture Notes in Computer Science, pages 39-51. Springer.

Fuhl, W., Santini, T. C., Kuebler, T., and Kasneci, E. (2015b). ElSe: Ellipse Selection for Robust Pupil Detection in Real-World Environments. arxiv: 1511.06575

Gidlöf, K., Wallin, A., Dewhurst, R., and Holmqvist, K. (2013). Using eye tracking to trace a cognitive process: Gaze behaviour during decision making in a natural environment. Journal of Eye Movement Research, 6(1):1-14.

Healy, A. F. and Proctor, R. W. (2003). Handbook of psychology: Experimental psychology.

Hillaire, S., Lecuyer, A., Cozot, R., and Casiez, G. (2008). Using an eye-tracking system to improve camera motions and depth-of-field blur effects in virtual environments. In Virtual Reality Conference, 2008. VR '08. IEEE, pages 47-50

Howard, I. P. (2012). Depth from accommodation and vergence. In Perceiving in DepthVolume 3 Other Mechanisms of Depth Perception, pages 1-14. Oxford University Press (OUP).

Kandel, E. R., Schwartz, J. H., and Jessell, T. M. (2000). Principles of neural science. McGraw-Hill, New York.

Kasneci, E., Kasneci, G., Kübler, T. C., and Rosenstiel, W. (2015). Online Recognition of Fixations, Saccades, and Smooth Pursuits for Automated Analysis of Traffic Hazard Perception. In Artificial Neural Networks, volume 4 of Springer Series in Bio-/Neuroinformatics, pages 411-434. Springer International Publishing.

Kasneci, E., Sippel, K., Heister, M., Aehling, K., Rosenstiel, W., Schiefer, U., and Papageorgiou, E. (2014). Homonymous visual field loss and its impact on visual exploration: A supermarket study. TVST, 3(6).

Kasthurirangan, S. (2014). Current methods for objectively measuring accommodation. Presented as AAO Workshop on Developing Novel Endpoints for Premium Intraocular Lenses.

Kourkoumelis, N. and Tzaphlidou, M. (2011). Eye safety related to near infrared radiation exposure to biometric devices. The Scientific World Journal, 11:520-528.
Lopes, P., Lavoie, R., Faldu, R., Aquino, N., Barron, J., Kante, M., and (advisor, W. M. (2012). Icraft eyecontrolled robotic feeding arm technology members.

Lukic, L., Santos-Victor, J., and Billard, A. (2014). Learning robotic eye-arm - hand coordination from human demonstration: A coupled dynamical systems approach. Biol. Cybern., 108(2):223-248.

Mulvey, F., Villanueva, A., Sliney, D., Lange, R., Cotmore, S., and Donegan, M. (2008). Exploration of safety issues in eyetracking. Technical Report IST-2003511598, COGAIN EU Network of Excellence.

Reichelt, S., Haussler, R., Fütterer, G., and Leister, N. (2010). Depth cues in human visual perception and their realization in 3D displays. In Three $\mathrm{Di}$ mensional Imaging, Visualization, and Display 2010, pages 76900B-76900B-12.

Rogers, A. (1988). Mosby's guide to physical examination. Journal of anatomy, 157:235.

Schaeffel, F., Wilhelm, H., and Zrenner, E. (1993). Interindividual variability in the dynamics of natural accommodation in humans: relation to age and refractive errors. The Journal of Physiology, 461(1):301320.

Shih, S.-W. and Liu, J. (2004). A novel approach to 3-d gaze tracking using stereo cameras. IEEE Transactions on Syst. Man and Cybern., part B, 34:234-245.

Sunday, D. (2012). Distance between 3d lines \& segments.

Świrski, L. and Dodgson, N. (2013). A fully-automatic, temporal approach to single camera, glint-free 3D eye model fitting. Proc. PETMEI.

Tafaj, E., Kasneci, G., Rosenstiel, W., and Bogdan, M. (2012). Bayesian online clustering of eye movement data. In Proceedings of the Symposium on Eye Tracking Research and Applications, ETRA '12, pages 285-288. ACM.

Wang, R. I., Pelfrey, B., Duchowski, A. T., and House, D. H. (2014). Online 3D Gaze Localization on Stereoscopic Displays. ACM Transactions on Applied Perception, 11(1):1-21 